\title{
Early Stage Strong Metal Support Interaction (SMSI) Effects In An Experimental Titania-Supported Platinum Catalyst: An Environmental TEM Study
}

\author{
C.E. Kliewer*, S. Miseo*, J.E. Baumgartner*, \\ E. Stach ${ }^{* \star *}$, and D. Zakharov*** \\ *ExxonMobil Research \& Engineering, Annandale, NJ 08801 \\ ${ }^{* * *}$ Purdue University, West Lafayette, IN 47907
}

Environmentally controlled TEM studies have long proven important in developing meaningful nanostructure/property relationship information. ${ }^{1}$ This is particularly true for many catalytic systems where small metal particle physical property data (e.g., melting point, freezing point, etc.) varies significantly from the bulk material. ${ }^{2}$ Thus, over time, various in-situ ${ }^{1,3-5}$ and ex-situ ${ }^{6-8}$ TEM techniques have been developed to more effectively characterize existing systems. These protocols, however, can also be strategically employed to improve the design of new catalytic systems and to more effectively control nanostructures under reactive conditions.

Standard wisdom often suggests that support materials are inactive at relatively low temperatures and that the primary concern in developing more active/selective materials involves parameters such as metal particle size and composition. However, $500{ }^{\circ} \mathrm{C} \mathrm{H}_{2}$ chemisorption studies conducted on $\mathrm{TiO}_{2}$-supported $\mathrm{Pt}$ particles revealed that it is a support effect (strong metal support interaction, SMSI) which leads to decreased metal particle $\mathrm{H}_{2}$ uptake over time and thus decreased catalytic activity. ${ }^{9-11}$

While both detailed conventional TEM (CTEM) $)^{12,13}$ and careful environmental TEM $(E T E M)^{14-16}$ investigations have substantiated the aforementioned chemisorption findings, recent ETEM studies conducted $\sim 200{ }^{\circ} \mathrm{C}$ under $\sim 1$ torr $\mathrm{H}_{2}$ have revealed unanticipated "early stage" SMSI effects in $\mathrm{Pt} / \mathrm{TiO}_{2}$. Pt metal acts as a catalyst and restructures the titania support (Figures 1a-1d). Electronic effects between the Pt and the restructured titania result in metal particle motion as well as metal particle tilting/faceting on the support. These previously undocumented effects result prior to formation of the $\mathrm{Ti}_{4} \mathrm{O}_{7}{ }^{12-13} \mathrm{SMSI}$ overlayer.

\section{ACKNOWLEDGEMENTS:}

The authors wish to thank, J.D. Kushnerick, M. Daage, and W.N. Delgass for coordinating ExxonMobil/Purdue University efforts, F. Riberio for ETEM gas handling system help, and E. Iglesia for insightful technical discussions.

\section{REFERENCES:}

1. E.P. Butler and K.F. Hale Dynamic Experiments in the Electron

Microscope (1981) Elsevier/North Holland Biomedical Press. 
2. P. Buffat and J.P. Borel, Phys. Rev. A 13 (1976) 228.

3. P.L. Gai, Microscopy \& Microanalysis 3 (1997) 617.

4. E.D. Boyes, Microscopy \& Microanalysis 3 (1997) 589.

5. P.A. Crozier, Microscopy \& Microanalysis 7 (2001) 1058.

6. I. Chan, J. Electron Microscopy Tech. (1985) 525.

7. L.F. Allard et. al., Proc. Microscopy \& Microanalysis 3 (1997) 595.

8. C.E. Kliewer et. al., Proc. Microscopy \& Microanalysis 1 (1999) 926.

9. S.J. Tauster, S.C. Fung, and R.L. Garten, JACS 100 (1978) 170.

10. S.J. Tauster and S.C. Fung, J. Catal. 55 (1978) 29.

11. S.J. Tauster et. al., Science 211 No. 4487 (1981) 1121.

12. R.T.K. Baker, E.B. Prestridge, and R.L. Garten, J. Catal. 56 (1979) 390.

13. R.T.K. Baker, E.B. Prestridge, and R.L. Garten, J. Catal. 59 (1979) 293.

14. E.D. Boyes et. al., Mat. Res. Soc. Sym. Proc., 404 (1996) 53

15. E.D. Boyes and P.L. Gai, Ultramicroscopy 67 (1997) 219.

16. P.L. Gai, Adv. Mater. 10 No. 15 (1998) 1259.

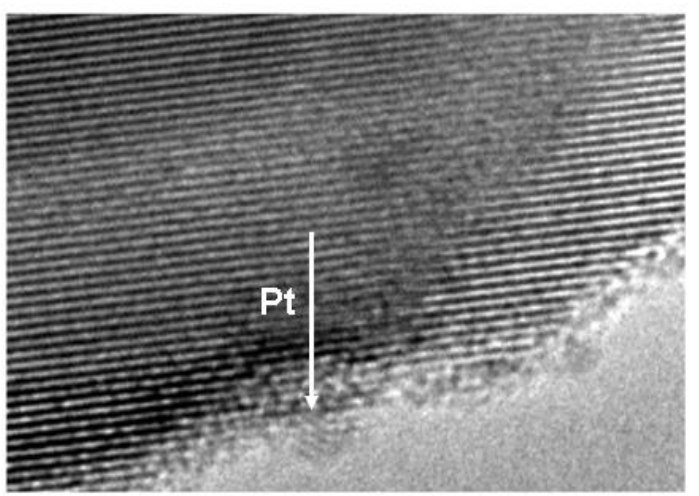

a

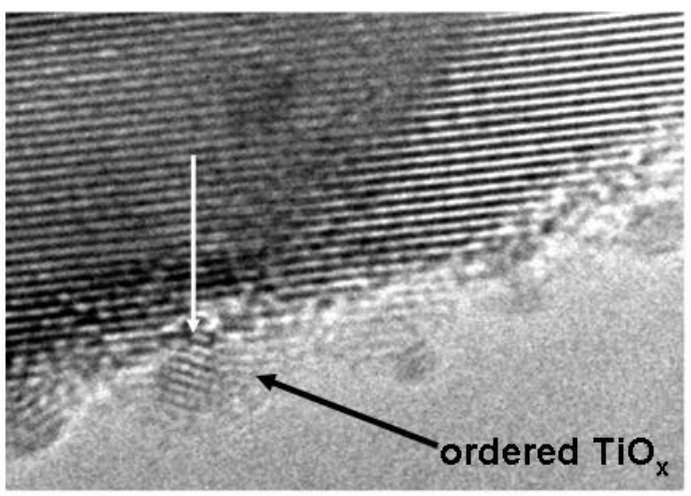

C

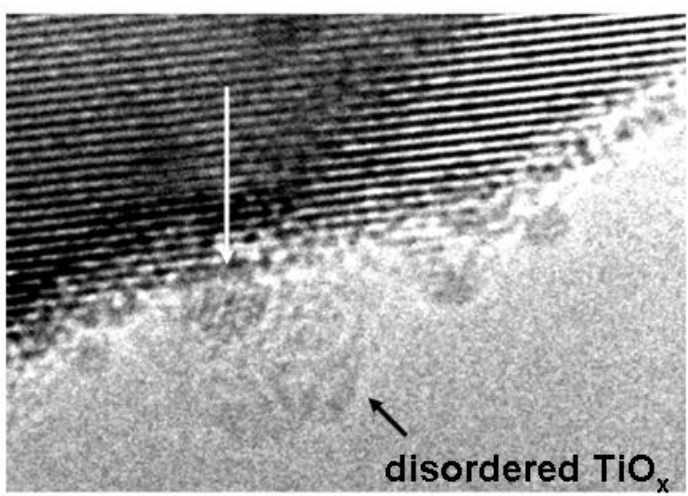

b

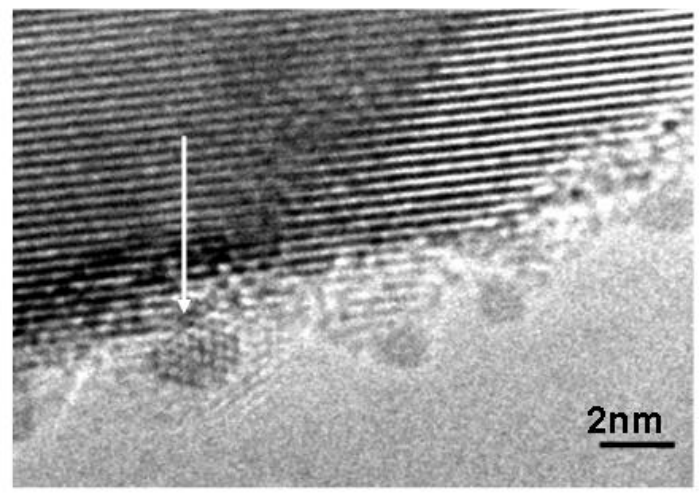

d

Figure 1: Bright field ETEM images showing $\mathrm{Pt} / \mathrm{TiO}_{2}$ catalyst at $\sim 200{ }^{\circ} \mathrm{C}$ under

$\sim 1$ torr $\mathrm{H}_{2}$ after (a) < $1 \mathrm{~min}$, (b) $\sim 1: 45 \mathrm{~min},(\mathrm{c}) \sim 3: 30 \mathrm{~min}$, and (d) $\sim 5 \mathrm{~min}$. 